\title{
Avaliação dos pacientes com Diabetes e Hipertensão em uma Estratégia de Saúde da Família localizada na zona rural do interior do Estado do Pará
}

\author{
Evaluation of patients with Diabetes and Hypertension in a Family Health Strategy located in the \\ countryside of the interior of the State of Pará \\ Evaluación de pacientes con Diabetes e Hipertensión en una Estrategia de Salud de la Familia \\ ubicada en el campo del interior del Estado de Pará
}

Recebido: 16/02/2021 | Revisado: 23/02/2021 | Aceito: 25/02/2021 | Publicado: 03/03/2021

\author{
Marcio Lima da Costa \\ ORCID: https://orcid.org/0000-0002-3717-5483 \\ Universidade Federal do Pará, Brasil \\ E-mail: marcio_limac@ hotmail.com \\ Rodolfo Botelho Moraes \\ ORCID: https://orcid.org/0000-0002-4523-4789 \\ Universidade Federal do Pará, Brasil \\ E-mail: rodolfobotelho3@gmail.com \\ Davis Wilker Nascimento Vaz \\ ORCID: https://orcid.org/0000-0002-0601-8194 \\ Universidade do Estado do Pará, Brasil \\ E-mail: daviswilkervaz@gmail.com \\ Gabriel Aguiar dos Santos \\ ORCID: https://orcid.org/0000-0002-0635-266X \\ Universidade do Estado do Pará, Brasil \\ E-mail: gab.ads96@gmail.com \\ Renan Corrêa da Costa Duarte \\ ORCID: https://orcid.org/0000-0002-4820-2117 \\ Universidade do Estado do Pará, Brasil \\ E-mail: duarterenan9@gmail.com \\ Jose Aufeu Barreto dos Santos Junior \\ ORCID: https://orcid.org/0000-0003-1848-8617 \\ Universidade Federal do Pará, Brasil \\ E-mail: aufeu.junior@hotmail.com \\ Thayssa Xavier de Figueiredo Menezes \\ ORCID: https://orcid.org/0000-0001-8806-732X \\ Centro Universitário do Estado do Pará, Brasil \\ E-mail: thayssamenezes@gmail.com \\ Roberto de Sousa Teixeira \\ ORCID: https://orcid.org/0000-0002-6513-403X \\ Universidade Federal do Pará, Brasil \\ E-mail: robertomed2009@hotmail.com
}

\begin{abstract}
Resumo
Objetivo: Avaliar os pacientes com diagnóstico de Diabetes Mellitus tipo 2 e Hipertensão Arterial Sistêmica em uma Estratégia de Saúde da Família (ESF) localizada na zona rural do município de Santa Bárbara do Pará, Estado do Pará, Brasil. Metodologia: Trata-se de um estudo epidemiológico, observacional, transversal e descritivo. A população estudada foi constituída pelos portadores de HAS e/ou DM II, atendidos na referida ESF, dentro do período de 1 ano. Resultados: 102 pessoas participaram do estudo. Desses, 70 pacientes eram hipertensos, 10 eram diabéticos e 22 possuíam HAS e DM II, concomitantemente. O gênero feminino foi o predominante com $57,84 \%$ dos casos. A etnia mais prevalente foi a parda (43,13\%). O estudo identificou uma baixa adesão à dieta recomendada, tanto em hipertensos $(21,42 \%)$ e diabéticos $(30 \%)$ quanto nos portadores das duas patologias $(45,45 \%)$. A taxa de adesão à prática regular de atividade física também foi baixa nos 3 grupos da presente pesquisa. A maior parte $(59,37 \%)$ dos diabéticos apresentou níveis glicêmicos acima do recomendado. A maioria (66,3\%) dos hipertensos também apresentou níveis pressóricos acima do adequado. Conclusão: Assim, é imperativo que haja um fortalecimento do vínculo da equipe multiprofissional da saúde com o paciente atendido na ESF, buscando sempre o exercício do cuidado em saúde de forma holística, com o intuito de melhorar a adesão ao tratamento da HAS e da DM II.
\end{abstract}

Palavras-chave: Diabetes Mellitus; Hipertensão; Estratégia saúde da família. 


\begin{abstract}
Objective: To evaluate patients diagnosed with Type 2 Diabetes Mellitus and Systemic Arterial Hypertension in a Family Health Strategy (FHS) located in the rural area of the municipality of Santa Bárbara do Pará, State of Pará, Brazil. Methodology: This is an epidemiological, observational, cross-sectional and descriptive study. The studied population consisted of patients with SAH and / or DM II, assisted in the referred FHS, within a period of 1 year. Results: 102 people participated in the study. Of these, 70 patients were hypertensive, 10 were diabetic and 22 had SAH and DM II, concomitantly. The female gender was predominant with $57.84 \%$ of cases. The most prevalent ethnic group was brown (43.13\%). The study identified low adherence to the recommended diet, both in hypertensive patients $(21.42 \%)$ and diabetics $(30 \%)$ and in patients with both pathologies $(45.45 \%)$. The rate of adherence to regular physical activity was also low in the 3 groups of the present study. Most $(59.37 \%)$ of diabetics had glycemic levels above the recommended. The majority $(66.3 \%)$ of hypertensive patients also had blood pressure levels above the appropriate level. Conclusion: Thus, it is imperative that there is a strengthening of the bond between the multidisciplinary health team and the patient assisted in the FHS, always seeking to exercise health care in a holistic way, in order to improve adherence to the treatment of SAH and DM II.
\end{abstract}

Keywords: Diabetes Mellitus; Hypertension; Family health strategy.

\title{
Resumen
}

Objetivo: Evaluar pacientes diagnosticados de Diabetes Mellitus tipo 2 e Hipertensión Arterial Sistémica en una Estrategia de Salud de la Familia (ESF) ubicada en el área rural del municipio de Santa Bárbara do Pará, Estado de Pará, Brasil. Metodología: Se trata de un estudio epidemiológico, observacional, transversal y descriptivo. La población estudiada estuvo constituida por pacientes con HSA y / o DM II, asistidos en el referido ESF, en un plazo de 1 año. Resultados: 102 personas participaron en el estudio. De estos, 70 pacientes eran hipertensos, 10 eran diabéticos y 22 tenían HSA y DM II de forma concomitante. Predominó el sexo femenino con el 57,84\% de los casos. El grupo étnico más prevalente fue el moreno (43,13\%). El estudio identificó una baja adherencia a la dieta recomendada, tanto en pacientes hipertensos $(21,42 \%)$ y diabéticos $(30 \%)$ como en pacientes con ambas patologías $(45,45 \%)$. La tasa de adherencia a la actividad física regular también fue baja en los 3 grupos del presente estudio. La mayoría $(59,37 \%)$ de los diabéticos tenían niveles glucémicos superiores a los recomendados. La mayoría (66,3\%) de los pacientes hipertensos también tenían niveles de presión arterial por encima del nivel apropiado. Conclusión: Por ello, es imperativo que se fortalezca el vínculo entre el equipo de salud multidisciplinario y el paciente atendido en la ESF, buscando siempre ejercer la atención de la salud de manera integral, con el fin de mejorar la adherencia al tratamiento de la HAS y DM II.

Palabras clave: Diabetes Mellitus; Hipertensión; Estrategia de salud familiar.

\section{Introdução}

As Doenças Crônicas Não Transmissíveis (DCNT) são a principal causa de morbimortalidade no mundo. Dentre elas, a Hipertensão Arterial Sistêmica (HAS) e o Diabetes Mellitus tipo 2 (DM2) são as mais comuns. Tratam-se de moléstias que elevam consideravelmente o risco cardiovascular e afetam significativamente a qualidade de vida dos pacientes acometidos (Escócio et al., 2020; Lima et al., 2020).

O tratamento dessas doenças é bastante complexo sendo composto por medidas farmacológicas e não farmacológicas, como mudança de hábitos alimentares, prática de atividade física regular e mudança no estilo de vida. Logo, na busca por um controle terapêutico adequado, um bom vínculo do paciente com a equipe multiprofissional da saúde é fundamental (Corgozinho et al., 2020; Santos et al., 2021).

Muitos pacientes com DM II e HAS não seguem o tratamento proposto por diversos motivos, como o desconhecimento sobre as doenças e suas complicações, a falta de informação sobre a necessidade de mudança dos hábitos de vida, o uso irregular dos fármacos ou até mesmo o abandono do uso dos medicamentos por conta própria. Esses e outros fatores interferem fortemente no controle adequado dessas doenças (Ramos et al., 2021; Pereira et al., 2021).

Nesse contexto, a Atenção Primária à Saúde (APS) do Sistema Único de Saúde (SUS) possui um papel fundamental no controle da HAS e DM II, pois é justamente nesse nível de atenção em que é possível a criação de um vínculo mais fidedigno do paciente com a equipe multiprofissional da saúde. Assim, um melhor seguimento dos pacientes com HAS e DM II na APS, pode resultar em importantes melhorias na adesão terapêutica e, desse modo, diminuir o risco de complicações tanto agudas quanto crônicas relacionadas a essas doenças (Lopes et al., 2021; Dantas \& Roncalli, 2020). 
No Brasil, uma das grandes dificuldades da saúde pública é o tratamento das DCNT como a HAS e DM II nas áreas remotas e rurais. Tratam-se de lugares, em sua maioria, dotados de menos recursos socioeconômicos e de saúde. Ademais, os profissionais de saúde que atuam nessas localidades, muitas vezes, encontram-se em um contexto de relativo isolamento que, frequentemente, dificulta o exercício de um cuidado integral e adequado ao paciente com essas doenças (Oliveira et al., 2018; Silva et al., 2018).

Nessa perspectiva, realizou-se o presente estudo que teve como objetivo avaliar os pacientes com diagnóstico de Diabetes Mellitus tipo 2 e Hipertensão Arterial Sistêmica em uma Estratégia de Saúde da Família (ESF) localizada na zona rural do município de Santa Bárbara do Pará, Estado do Pará, Brasil.

\section{Metodologia}

Trata-se de um estudo epidemiológico, observacional, transversal e descritivo. A pesquisa foi realizada em uma ESF localizada na zona rural do município de Santa Bárbara do Pará, Estado do Pará, Brasil. A população estudada foi constituída pelos portadores de HAS e/ou DM II, atendidos na referida ESF, dentro do período de 1 ano.

Foram incluídos na presente pesquisa os pacientes matriculados na ESF que se enquadravam nas seguintes categorias: (1) pacientes com Diabetes, independente da faixa etária e do gênero; (2) pacientes com Hipertensão, independente da faixa etária e do gênero; (3) pacientes com Diabetes e Hipertensão, independente da faixa etária e do gênero.

A coleta de dados foi feita mediante a análise dos prontuários dos pacientes matriculados na ESF. Dispuseram-se como variáveis epidemiológicas analisadas no presente estudo: idade, área de residência (rural ou urbana), gênero, etnia, nível de escolaridade e o perfil clínico.

Os resultados obtidos foram analisados mediante o uso do programa Biostat 5.0, sendo empregado o teste Quiquadrado, considerando serem significativos os dados com $\mathrm{p}<0,05$, para haver um intervalo de confiança de $95 \%$. Tabelas e gráficos foram confeccionados utilizando o programa Microsoft Office Excel 2013 e a editoração do texto foi realizada através do Microsoft Office Word 2013.

A pesquisa não apresentou riscos à população de estudo, uma vez que a coleta de dados foi feita através da análise de prontuários. Os pesquisadores asseguraram a confidencialidade, a privacidade e não estigmatização dos pacientes. Foi mantido o anonimato da identificação e das informações pessoais relacionadas à amostra investigada, de acordo com a Resolução 466/2012 do Conselho Nacional de Saúde (CNS).

Destaca-se que a coleta de dados teve início apenas após a aprovação do projeto pelo Comitê de Ética em Pesquisa (CEP) do Instituto de Ciências da Saúde da Universidade Federal do Pará (UFPA), em Belém - PA.

\section{Resultados e Discussão}

No total, 102 pessoas participaram do estudo. A Figura 1 demonstra que 70 pacientes eram hipertensos, 10 eram diabéticos e 22 possuíam HAS e DM II, concomitantemente. Esta predominância numérica de hipertensos sobre diabéticos é bem consolidada na literatura. Segundo a Organização Mundial da Saúde (OMS), 20 a 40\% da população adulta possui HAS. Com relação à DM, essa prevalência é de uma pessoa a cada dez (Tortorella et al., 2017; Fiório et al., 2020). 
Figura 1 - Distribuição dos participantes da pesquisa de acordo com o diagnóstico de HAS, DM II e HAS/DM II na ESF.

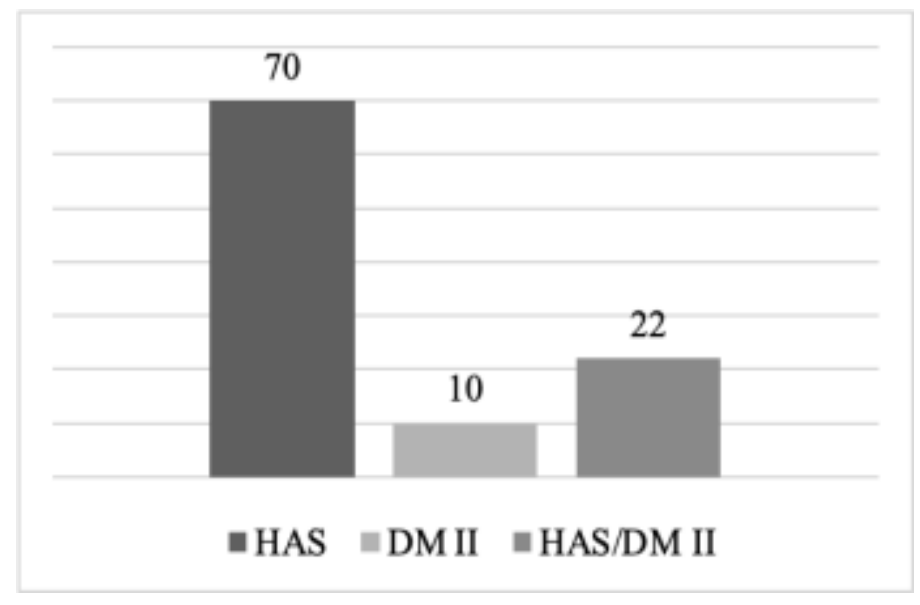

Fonte: Protocolo de pesquisa.

A HAS e DM II associadas possuem um potencial ainda maior de morbidade e mortalidade. Aumentam significativamente o risco cardiovascular e predispõe o paciente ao surgimento de importantes complicações como doença renal, doença coronariana, insuficiência cardíaca e acidente vascular encefálico (Francisco et al., 2018; Prates et al., 2020).

Com relação ao gênero, o feminino foi predominante, sendo responsável por 57,84\% dos casos (Tabela 1). Prates et al. (2020), ao analisar as variáveis sociodemográficas dos pacientes com HAS/DM II em uma Estratégia de Saúde da Família (ESF) no interior de Minas Gerais, também que o sexo feminino foi responsável pela maioria dos casos (70\%). No estudo de Queiroz et al., (2020) realizado no estado de Goiás, 64\% das pacientes com HAS e DM, eram diabéticas.

Tabela 1 - Aspectos sociodemográficos dos pacientes hipertensos, diabéticos e diabéticos/hipertensos assistidos na ESF.

\begin{tabular}{|c|c|c|c|c|c|}
\hline Variáveis & HAS & $\begin{array}{c}\mathrm{HAS} / \mathrm{DM} \\
\text { II }\end{array}$ & DM II & Total & $\%$ \\
\hline \multicolumn{6}{|l|}{ Gênero } \\
\hline Masculino & 32 & 8 & 3 & 43 & $42,15 \%$ \\
\hline Feminino & 38 & 14 & 7 & 59 & $\mathbf{5 7 , 8 4} \%$ \\
\hline \multicolumn{6}{|l|}{ Etnia } \\
\hline Parda & 26 & 12 & 6 & 44 & $43,13 \%$ \\
\hline Negra & 12 & 4 & 0 & 16 & $15,6 \%$ \\
\hline Branca & 13 & 1 & 0 & 14 & $13,72 \%$ \\
\hline Não registrado & 19 & 5 & 4 & 28 & $27,45 \%$ \\
\hline \multicolumn{6}{|l|}{ Escolaridade } \\
\hline Analfabetos & 8 & 4 & 1 & 13 & 12,74 \\
\hline $\begin{array}{l}\text { Fundamental } \\
\text { incompleto }\end{array}$ & 20 & 5 & 4 & 29 & $28,43 \%$ \\
\hline $\begin{array}{l}\text { Fundamental } \\
\text { completo }\end{array}$ & 7 & 3 & 1 & 11 & $10,78 \%$ \\
\hline Médio incompleto & 4 & 2 & 0 & 6 & $5,88 \%$ \\
\hline Médio completo & 10 & 1 & 0 & 11 & $10,78 \%$ \\
\hline Superior & 1 & 0 & 0 & 1 & $0,98 \%$ \\
\hline Não registrado & 20 & 7 & 4 & 21 & $3,92 \%$ \\
\hline
\end{tabular}


O estudo identificou uma baixa adesão à dieta (Figura 2) recomendada, tanto em hipertensos $(21,42 \%)$ e diabéticos $(30 \%)$ quanto nos portadores das duas patologias $(45,45 \%)$. Diversos estudos afirmam que, no geral, tanto os pacientes com HAS e quando com DM, apresentam uma baixa adesão ao tratamento não medicamentoso (Stopa et al., 2018; Albarello et al., 2020).

Figura 2 - Adesão à dieta dos pacientes hipertensos, diabéticos e diabéticos/hipertensos assistidos na ESF.

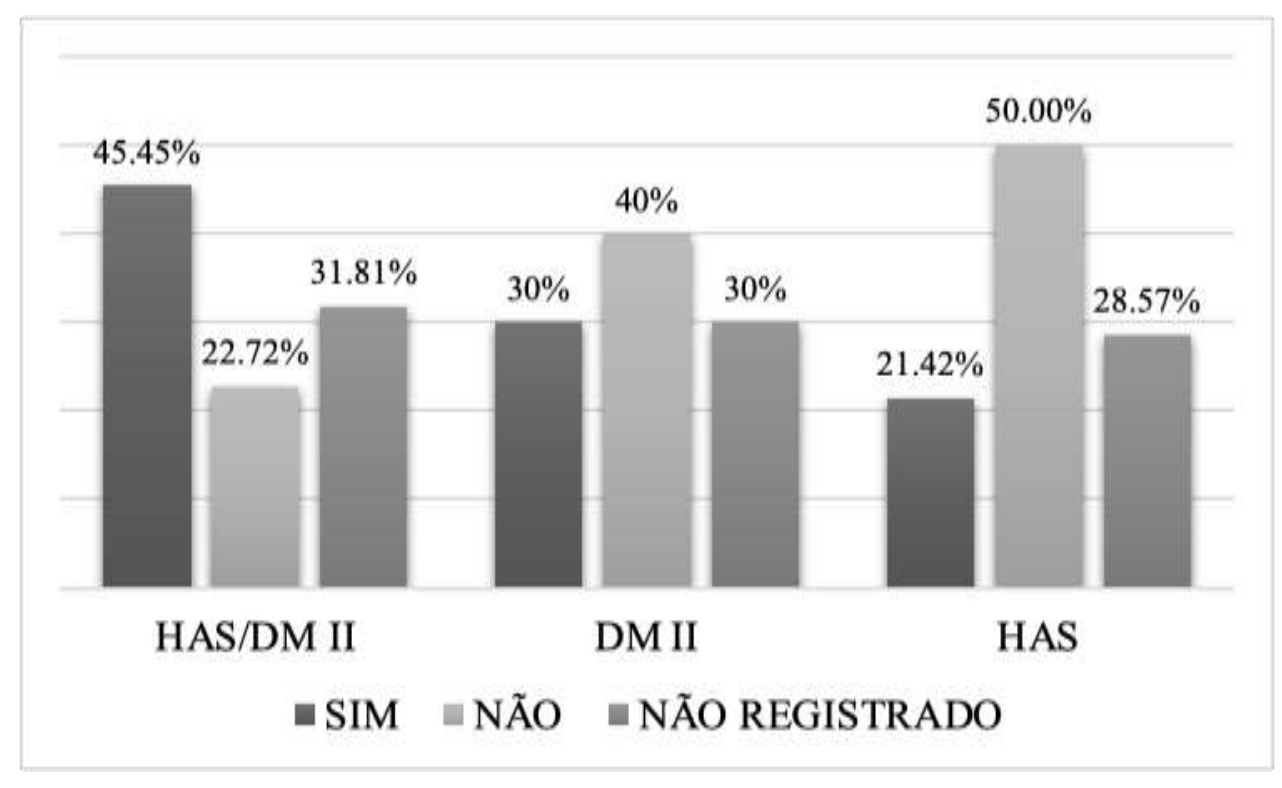

Fonte: Protocolo de pesquisa.

Diversos são os fatores que dificultam a adesão dos pacientes a uma dieta saudável, como é recomendado para os pacientes com HAS e DM. Dentre esses destacam-se fatores financeiros, devido ao alto preço dos alimentos saudáveis em contrapartida com o baixo valor e alta facilidade de acesso a produtos maléficos à saúde; a falta de conhecimento sobre a composição dos alimentos, fazendo com que, muitas vezes, o paciente associe a piora das doenças apenas com a alta ingestão de açúcar e sal, sem levar em consideração os outros componentes prejudiciais à saúde e aspectos culturais, como a dificuldade de fracionar a alimentação devido ao hábito consolidado de realizar apenas as três refeições principais (Maeyama et al., 2020; Macete \& Borges, 2020).

Com relação à prática de atividades físicas regulares (Figura 3), a taxa de adesão também foi baixa nos 3 grupos da presente pesquisa. Os principais fatores dificultadores da prática diária dos exercícios físicos apontados pelos estudos são a falta a tempo, cansaço, falta de acompanhante, o longo percurso até os locais que orientam a prática de exercícios físicos e a escassez de locais públicos com infraestrutura adequada para a realização dessas atividades (Liz \& Andrade, 2016). 
Figura 3 - Adesão à atividade física programada dos pacientes hipertensos, diabéticos e diabéticos/hipertensos assistidos na ESF.

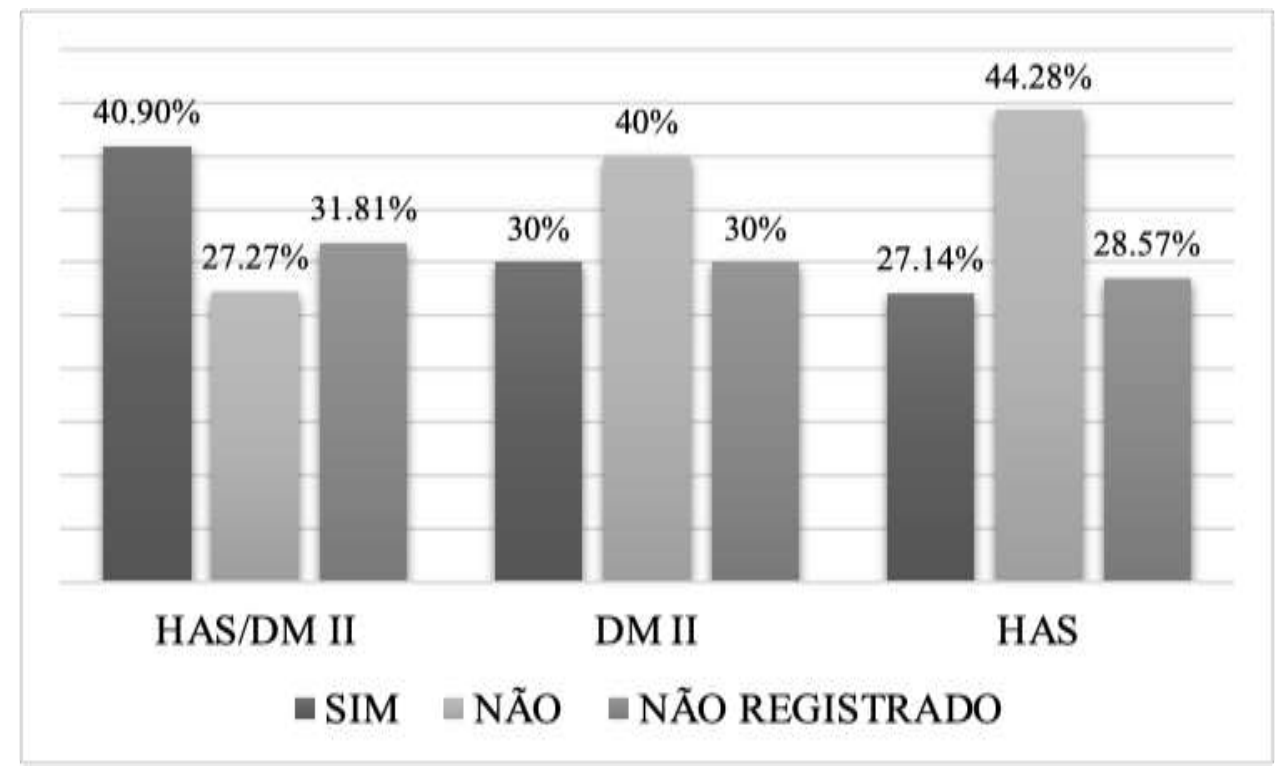

Fonte: Protocolo de pesquisa.

Quanto ao controle laboratorial anual da glicemia de jejum dos diabéticos e dos pacientes com DM II e HAS ao mesmo tempo, a Tabela 2 mostra que a maior parte $(59,37 \%)$ apresentou níveis glicêmicos acima do recomendado (maior do que $130 \mathrm{mg} / \mathrm{dl}$ ). Esses dados são preocupantes pois um controle inadequado da glicemia em pacientes diabéticos associa-se ao aumento de complicações tanto agudas como hipoglicemia, estado hiperglicêmico hiperosmolar e cetoacidose diabética, quanto crônicas, como retinopatia, neuropatias, doença renal crônica, dentre outras (Cortez et al., 2015).

Tabela 2 - Controle laboratorial anual da glicemia de jejum dos pacientes diabéticos/hipertensos e apenas diabéticos, assistidos na ESF.

\begin{tabular}{lcc}
\hline \multicolumn{1}{c}{ Variáveis } & $\begin{array}{c}\text { DM II e DM } \\
\text { II/HAS }\end{array}$ & $\%$ \\
\hline Glicemia de jejum & & \\
$\begin{array}{l}\text { Entre } 70 \mathrm{mg} / \mathrm{dl}-130 \\
\mathrm{mg} / \mathrm{dl}\end{array}$ & 8 & $25 \%$ \\
Acima de $130 \mathrm{mg} / \mathrm{dl}$ & 19 & $59,37 \%$ \\
Não realizaram & 5 & $15,62 \%$ \\
\hline
\end{tabular}

Fonte: Protocolo de pesquisa.

Com relação ao nível de pressão arterial dos hipertensos e dos pacientes com HAS e DM II concomitantemente, a Figura 4 mostra que a maioria (66,3\%) apresentou níveis pressóricos acima do adequado (140 x $90 \mathrm{mmHg}$ ), indicando que, provavelmente, há uma baixa adesão ao tratamento da HAS na região estudada. 
Figura 4 - Classificação quanto ao nível da pressão arterial, medida na última consulta, dos pacientes hipertensos e hipertensos/diabéticos, assistidos na ESF.

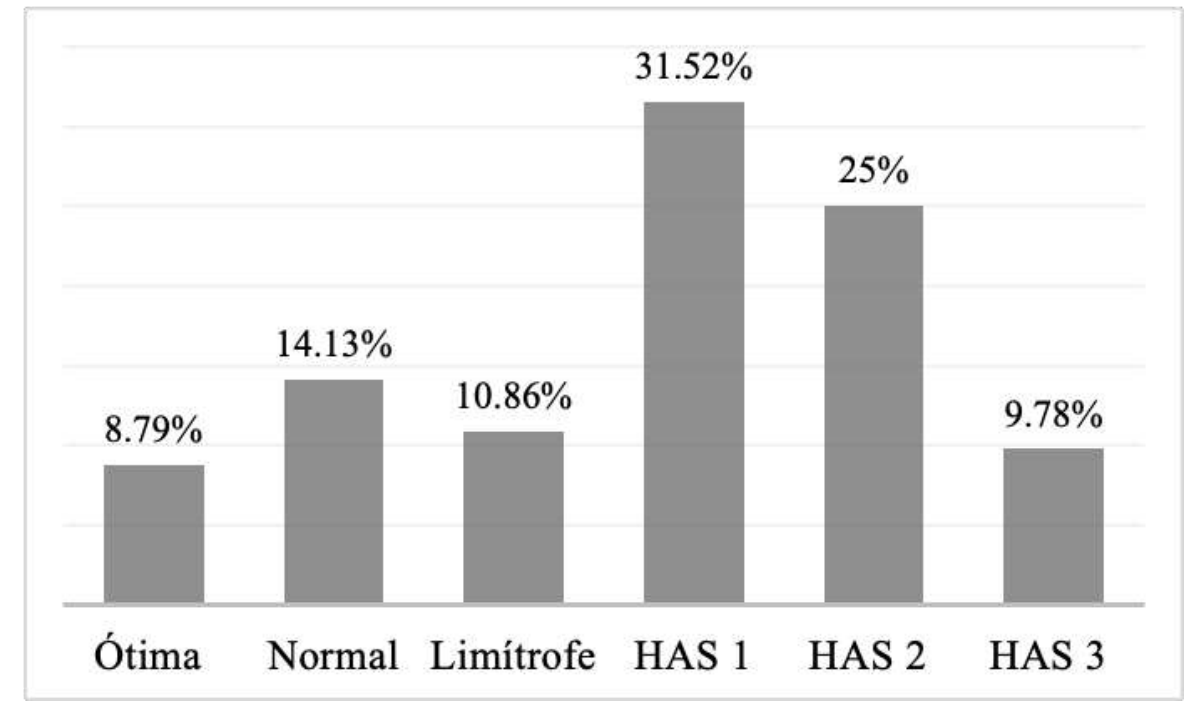

Fonte: Protocolo de pesquisa.

Diversos são os fatores que dificultam a adesão correta ao tratamento da HAS na ESF. Dentre esses têm-se aspectos sociodemográficos como o baixo nível socioeconômico e de escolaridade de boa parte dos pacientes atendidos pela ESF; fatores relacionados ao uso do medicamento tais como a utilização de múltiplos fármacos no mesmo dia, esquecimento do uso dos anti-hipertensivos, desconhecimento acerca dos possíveis efeitos colaterais relacionados a essas medicações e até mesmo problemas estruturais, como a falta de medicações em postos de saúde e problemas no agendamento de consultas (Oliveira et al., 2021).

\section{Conclusão}

O estudo identificou que a HAS foi mais prevalente do que a DM II na região analisada. Também se notou que uma porção considerável dos pacientes portavam HAS e DM II, concomitantemente. Dentre os pacientes estudados, houve um predomínio do gênero feminino e da etnia parda.

Ao analisar os dados obtidos, verificou-se que houve uma baixa adesão aos dois principais pilares do tratamento não medicamentoso dessas doenças: dieta saudável e a prática regular de atividade física. Com relação ao controle glicêmico dos pacientes diabéticos analisados pelo estudo, a maior parte apresentou níveis glicêmicos acima do recomendado para a DM II. Os hipertensos, por sua vez, também apresentaram, em sua maioria, níveis de pressão arterial maiores do que o adequado para o controle da doença.

Nessa perspectiva, é necessário que a prevenção contra a HAS e DM II seja prioridade na ESF. Assim, faz-se necessário um maior empenho dos profissionais de saúde em fornecer orientação e incentivo aos pacientes atendidos, de modo a estimular a mudança de hábitos de vida antes mesmo do diagnóstico de HAS e DM II, principalmente para os pacientes com maior predisposição ao desenvolvimento dessas doenças. Ademais, é imperativo que haja um fortalecimento do vínculo da equipe multiprofissional da saúde com o paciente atendido na ESF, buscando sempre o exercício do cuidado em saúde de forma holística, considerando as individualidades e as condições socioeconômicas de cada um, com o intuito de melhorar a adesão ao tratamento da HAS e da DM II. 


\section{Referências}

Albarello, J., Santos, A. D. L., Gesuino, D. B., Medeira, K., \& Ferraz, F. (2020). Adesão ao tratamento da hipertensão arterial sistêmica de pacientes participantes do programa hiperdia de uma estratégia em saúde da família. Saúde (Santa Maria), 46(2): e48245.

Corgozinho, M. L. M. V., Lovato, A., Martins, I. C. F., Mota, A. P. L., \& Mendes, A. C. R. (2020). Educação em diabetes e mudanças nos hábitos de vida. Research, Society and Development, 9(3), e175932566.

Cortez, D. N., Reis, I. A., Souza, D. A. S., Macedo, M. M. L., \& Torres, H. C. (2015). Complicações e o tempo de diagnóstico do diabetes mellitus na atenção primária. Acta Paulista de Enfermagem, 28(3), 250-255.

Dantas, R. C. O., \& Roncalli, A. G. (2020). Reprodutibilidade do protocolo para usuários com hipertensão arterial assistidos na Atenção Básica à Saúde. Ciência \& Saúde Coletiva, 25(8), 3037-3046.

Escócio, E. M. S., Aguiar, A. P., Silva, A. L., Canto, T. G., Pereira, P. M. P., \& Silva, C. D. L. (2020). Perfil clínico e fatores de risco cardiovasculares em policiais militares do município de Santarém, Oeste do Pará. Research, Society and Development, 9(8), e517985737.

Fiório, C. E., Cesar, C. L. G., Alves, M. C. G. P., Goldbaum, M. (2020). Prevalência de hipertensão arterial em adultos no município de São Paulo e fatores associados. Revista Brasileira de Epidemiologia, 23: E200052.

Francisco, M. S. B., Segri, N. J., Borim, F. S. A., \& Malta, D. C. Prevalência simultânea de hipertensão e diabetes em idosos brasileiros: desigualdades individuais e contextuais. Ciência \& Saúde Coletiva, 23 (11): 3829-3840.

Lima, D. C., Garcia, M. P., Lima, E. S., \& Bezerra, C. C. (2020). Educação em saúde como ferramenta na prevenção de doenças cardiovasculares no Programa de Atenção à Saúde do Idoso. Research, Society and Development, 9(10), e079107382.

Liz, C. M., \& Andrade, A. (2016). Análise qualitativa dos motivos de adesão e desistência da musculação em academias. Revista Brasileira de Ciências Do Esporte, 38(3), 267-274.

Lopes, M. S., Justino, D. C. P., \& Andrade, F. B. (2021). Assistência à saúde na atenção primária aos portadores de hipertensão arterial sistêmica e diabetes mellitus. Revista Ciência Plural, 7(1), 40-56.

Macete, K. G., \& Borges, G. F. (2020). Não Adesão ao Tratamento não Medicamentoso da Hipertensão Arterial Sistêmica. Revista Saúde em Foco, 7(1), 128154.

Maeyama, M. A., Pollheim, L. C. F., Wippel, M., Machado, C., \& Veiga, M. V. (2020). Aspectos relacionados à dificuldade do controle glicêmico em pacientes com Diabetes Mellitus tipo 2 na Atenção Básica. Brazilian Journal of Development, 6(7), 47352-47369.

Oliveira, A. R., Sousa, Y. G., Diniz, I. V. A., Medeiros, S. M., Martiniano, C. \& Alves, M. (2019). O cotidiano de enfermeiros em áreas rurais na estratégia saúde da família. Revista Brasileira de Enfermagem, 72(4), 970-977.

Oliveira, J. R. A., Filho, M. A. A. S., Santos, M. J. M. N., Militão, T. O., \& Santana, A. G. C. (2021). Fatores de risco para baixa adesão ao tratamento farmacológico de hipertensão arterial sistêmica na Atenção Primária à Saúde. Revista Eletrônica Acervo Saúde, 13(1), e5514.

Pereira, I. S., Santos, M. A., Sousa, M. T., Fonseca, H. A. T., Pereira, M. L., Virgens, C. M. B., Carvalho, J. S. M., \& Carvalho, F. L. Q. (2021). Avaliação da não adesão ao tratamento farmacológico da hipertensão arterial sistêmica em uma população de Salvador-BA. Brazilian Journal of Development, 7(1), 153174.

Prates, E. J. S., Souza, F. L. P., Prates, M. L. S., Moura, J. P., \& Carmo, T. M. D. (2020). Características clínicas de clientes com hipertensão arterial e diabetes mellitus. Revista de Enfermagem UFPE online, 14:e244110.

Queiroz, L. F., Fontana, A. P., Rodrigues, B. T., Filho, P. A., Marques, M. A., Mendonça, J. P., \& Essado, A. V. M. (2020). Perfil epidemiológico de portadores de hipertensão arterial sistêmica e de diabetes mellitus do bairro martins no município de Rio Verde/GO. Brazilian Journal of Development, 6(11), $87113-87126$

Ramos, C. H. S., Adeodato, A. G., Costa, N., Lima, D. J. M., Pereira, J. L. D., \& Silva, N. A. (2021). Atuação do enfermeiro na identificação dos fatores associados à não adesão ao idoso no tratamento de hipertensão arterial sistêmica. Research, Society and Development, 10(1), e50510111863.

Santos, G. O., Santos, L. L., Silva, D. N., \& Silva, S. L. (2020). Exercícios físicos e diabetes mellitus: Revisão. Brazilian Journal of Development, 7(1), 88378847 .

Silva, E. M., Portela, R. A., Medeiros, A. L. F., Cavalcante, M. C. W., \& Costa, R. T. A. (2018). Os desafios no trabalho da enfermagem na estratégia saúde da família em área rural: revisão integrativa. Hygeia, 14(28), 1-12.

Stopa, S. R., Cesar, C. L. G., Segri, N. J., Alves, M. C. G. P., Barros, M. B. A., \& Goldbaum, M. (2018). Prevalência da hipertensão arterial, do diabetes mellitus e da adesão às medidas comportamentais no Município de São Paulo, Brasil, 2003-2015. Cadernos de Saúde Pública, 34 (10):e00198717.

Tortorella, C. C. S., Corso, A. C. T., Chica, D. A. G., \& Melhen, A. R. F. (2017). Tendência temporal da prevalência de hipertensão arterial sistêmica e diabetes mellitus entre adultos cadastrados no Sistema Único de Saúde em Florianópolis, Santa Catarina, 2004-2011*. Revista Epidemiologia e Serviços de Saúde, 26(3), 469-480. 